\title{
Solving fully neutrosophic linear programming problem with application to stock portfolio selection
}

\author{
Hamiden Abd El-Wahed Khalifa ${ }^{1}$ and Pavan Kumar ${ }^{2, *}$ \\ ${ }^{1}$ Operations Research Department, Faculty of Graduate Studies for Statistical Research \\ Cairo University, Giza, Egypt \\ E-mail:〈hamiden@cu.edu.eg \\ 1 Mathematics Department, College of Science and Arts \\ Al-Badaya, Qassim University, Saudi Arabia \\ E-mail: 〈Ha.Ahmed@qu.edu.sa〉 \\ ${ }^{2}$ Mathematics Division, School of Advanced Science and Languages \\ VIT Bhopal University Sehore, MP-466114, India \\ E-mail: 〈pavankmaths@gmail.com〉
}

\begin{abstract}
Neutrosophic set is considered as a generalized of crisp set, fuzzy set, and intuitionistic fuzzy set for representing the uncertainty, inconsistency, and incomplete knowledge about the real world problems. In this paper, a neutrosophic linear programming (NLP) problem with single-valued trapezoidal neutrosophic numbers is formulated and solved. A new method based on the so-called score function to find the neutrosophic optimal solution of fully neutrosophic linear programming (FNLP) problem is proposed. This method is more flexible than the linear programming (LP) problem, where it allows the decision maker to choose the preference he is willing to take. A stock portfolio problem is introduced as an application. Also, a numerical example is given to illustrate the utility and practically of the method.
\end{abstract}

Keywords: linear programming, neutrosophic programming, single-valued trapezoidal neutrosophic numbers, score function, stock profit

Received: April 23, 2020; accepted: October 05, 2020; available online: December 18, 2020

DOI: 10.17535 /crorr.2020.0014

\section{Introduction}

Linear programming (LP) is an important tool used by decision maker (DM). This is applied frequently in real-world applicable problems. In spite of having a vast decision making experience, the decision maker cannot always articulate the goals precisely. Decision-making in a fuzzy environment, developed by Bellman and Zadeh [4] improved and a great help in the management decision problems. The fuzzy set theory and its applications and fuzzy programming with several objective functions were proposed by Zimmermann [47]. Many researchers adopted this concept for solving fuzzy linear programming (FLP) problems like Campos \& Verdegay [6], Ebrahimnejad et al. [10], and Ganesan \& Veeramani [12]

Fuzzy linear programming (FLP) is LP in which the parameters or decision variables are represented by fuzzy numbers. Tanaka et al. [38] and Zimmermann [47] are the pioneers of FLP. Many approaches for FLP problem have taken in consideration [5, 19, 26, 28, 29, 35, 43, 44, 46]. Khalifa et al. [23] studied the neutrosophic complex programming problem, and determined its

${ }^{*}$ Corresponding author. 
optimal solution by using lexicographic order. There are several ways for solving this problem $[2,8,11,14,15,17,2,25,33,34,36,37]$.

Portfolio selection (PS) problem is equivalent to the investor selecting the optimal portfolio from a set of possible portfolios Also, it focuses on the optimal allocation of one's wealth to obtain maximum profitable return under minimum risk control as discussed by Gao and Liu [13]. Markowitz [30] was the pioneer of the Modern Portfolio Theory. Gao and Liu [13] based on the theory of uncertainty to develop a risk-free protection index model for PS. Liu and Qin [27] investigated a mean semi-absolute deviation model for uncertain. Zhu [45] developed a mean semi-absolute deviation model for uncertain PS. A portfolio-adjusting problem was proposed by Huang and Ying [16]. Dutta and Kumar [9] presented an application of fuzzy goal programming approach to multi objective linear fractional inventory model. Kumar [24] studied an inventory model following the concept of salvage value to determine the optimal inventory policy. Prameela and Kumar [32] studied the single transmit fuzzy queuing model with two-classes by using ranking technique.

A generalization of the intuitionistic set, classical set, fuzzy set, paraconsistent set, dialetheist set, paradoxist set, tautological set based on neutrosophy. Neutrosophic theory was introduced by Smarandache as a tool to handle undetermined information. Hussein et al. [18] studied a neutrosophic LP problem, where they converted the neutrosophic model into the corresponding crisp based on the neutrosophic set parameters. Abdel- Basset et al. [1] introduced a technique for solving Lp problem with all of parameters represented by trapezoidal neutrosophic numbers. Nafei et al. [31] studied fully neutrosophic LP problem, where all the parameters are represented by triangular interval- valued neutrosophic numbers. They used the ranking function to convert the neutrosophic model into its crisp one, and then applied the standard methods for obtaining the solution. Darehmiraki [7] developed a new ranking method for solving the LP problem involving neutrosophic numbers in all the coefficients of the objective function and constraints.

Portfolio investment (PI) is quoted securities investment, a narrow sense of investment. It refers to the behavior that an enterprise or individual buys negotiable securities such accumulated money to earn profits. Wang et al. [40] studied the single valued sets in neutrosophic environment. Portfolio investment is mainly composed of three elements: income, risk and time as described by Yin [41].

This paper attempts to formulate and solve the linear programming (LP) problem in a neutrosophic environment. The problem is considered by incorporating for single valued trapezoidal neutrosophic number. A new method to find the neutrosophic optimal solution of FNLP problem is proposed.

The outlay of the paper is organized as follows: In Section 2 basic concepts and results related to fuzzy numbers, trapezoidal fuzzy numbers, intuitionistic trapezoidal fuzzy numbers, and neutrosophic set are recalled. In Section 3, fully neutrosophic linear programming (FNLP) problem is formulated and a solution method for obtaining the solution is introduced. Section 4 introduces a stock portfolio problem as an application and two examples for illustration are presented. Finally, some concluding remarks are reported in Section 5.

\section{Preliminaries}

In order to discuss our problem conveniently, basic concepts and results related to fuzzy numbers, trapezoidal fuzzy numbers, intuitionistic trapezoidal fuzzy numbers, and neutrosophic set are recalled.

Definition 1 (Zadeh [42]). A fuzzy set $\tilde{A}$ defined on the set of real numbers $\Re$ is said to be fuzzy number, if its membership function $\mu_{\tilde{A}}: \Re \rightarrow[0,1]$ hs the following properties:

(i) $\tilde{A}$ is an upper semi-continuous membership function; 
(ii) $\tilde{A}$ is convex,i. e., $\mu_{\tilde{A}}(\lambda x+(1-\lambda)) \geq \min \left\{\mu_{\tilde{A}}(x), \mu_{\tilde{A}}(y)\right\}, \lambda \in[0,1]$, for all $x, y \in \Re$;

(iii) $\tilde{A}$ is normal, $i$. e., there exists $x_{0} \in \Re$ for which $\mu_{\tilde{A}}\left(x_{0}\right)=1$;

(iv) $\operatorname{Supp}(\tilde{A})=\left\{x \in \Re: \mu_{\tilde{A}}(x)>0\right\}$ is the support of the $\tilde{A}$, and its closure $\operatorname{cl}(\operatorname{supp}(\tilde{A})$ is compact set.

Definition 2 (Kaufmann and Guta [21]). A fuzzy number $\tilde{A}\left(a_{1}, a_{2}, a_{3}, a_{4}\right)$ on $\Re$ is a trapezoidal fuzzy number if its membership function $\mu_{\tilde{A}}(x): \Re \rightarrow[0,1]$ has the following characteristics set:

$$
\mu_{\tilde{A}}(x)= \begin{cases}\frac{x-a_{1}}{a_{2}-a_{1}}, & a_{1} \leq x \leq a_{2} \\ 1, & a_{2} \leq x \leq a_{3} \\ \frac{a_{4}-x}{a_{4}-a L 3} & a_{3} \leq x \leq a_{4} \\ 0, & \text { elsewhere }\end{cases}
$$

Definition 3 (Atanassov [3]). Let $M$ be a nonempty set. An intuitionistic fuzzy set $\tilde{A}_{I}$ of $M$ is defined as $\tilde{A}_{I}=\left\{\left\langle x, \vartheta_{\tilde{A}_{I}}(x), \rho_{\tilde{A}_{I}}(x): x \in X\right\rangle\right\}$, where $\vartheta_{\tilde{A}_{I}}(x)$ and $\rho_{\tilde{A}_{I}}(x)$ are membership and nonmembership function, respectively such that $\vartheta_{\tilde{A}_{I}}(x), \rho_{\tilde{A}_{I}}(x): M \rightarrow[0,1]$ and $0<\vartheta_{\tilde{A}_{I}}(x)+$ $\rho_{\tilde{A}_{I}}(x) \leq 1$, for all $x \in M$.

Definition 4 (Atanassov [3]). An intuitionistic fuzzy subset $\tilde{A}_{I}=\left\{\left\langle x, \vartheta_{\tilde{A}_{I}}(x): x \in M\right\rangle\right\}$ of $\Re$ is called an intuitionistic fuzzy number if the following conditions hold:

(i) There exists $m \in \Re$ such that $\vartheta_{\tilde{A}_{I}}(m)=1$, and $\rho_{\tilde{A}_{I}}(m)=0$,

(ii) $\vartheta_{\tilde{A}_{I}}$ is continuous function from $\Re \rightarrow[0,1]$ such that $0 \leq \vartheta_{\tilde{A}_{I}}(x)+\rho_{\tilde{A}_{I}} \leq 1$, for all $x \in M$, and

(iii) The membership and non-membership functions of $\tilde{A}_{I}$ are:

$$
\begin{gathered}
\vartheta_{\tilde{A}_{I}}(x)= \begin{cases}0, & -\infty<x<\infty ; \\
h(x), & a_{1} \leq x \leq a_{2} ; \\
l(x) & a_{2} \leq x \leq a_{3} ; \\
0, & a_{3} \leq x<\infty .\end{cases} \\
\rho_{\tilde{A}_{I}}(x)= \begin{cases}0, & -\infty<x \leq a_{1}^{\circ} ; \\
h^{*}(x), & a_{1}^{\circ} \leq x \leq a_{2} ; \\
l^{*}(x) & a_{2} \leq x \leq a_{3}^{\circ} ; \\
0, & a_{3}^{\circ} \leq x<\infty .\end{cases}
\end{gathered}
$$

where, $h, h^{*}, l, l^{*}$ are functions from $\Re \rightarrow[0,1], h$, and $l^{*}$ are strictly increasing functions, and $l$, and $h^{*}$ are strictly decreasing functions with $0 \leq h(x)+h^{*}(x) \leq 1$, and $0 \leq$ $l(x)+l^{*}(x) \leq 1$.

Definition 5 (Jianqiang and Zhong [20]). A trapezoidal intuitionistic fuzzy number is denoted by $\tilde{A}_{I T}=\left\langle\left(a_{1}, a_{2}, a_{3}, a_{4}\right),\left(a_{1}^{\circ}, a_{2}, a_{3}, a_{4}^{\circ}\right)\right\rangle$, where $a_{1}^{\circ} \leq a_{1} \leq a_{2} \leq a_{3} \leq a_{4} \leq a_{4}^{\circ}$, with membership and non-membership functions are:

$$
\vartheta_{\tilde{A}_{I T}}(x)= \begin{cases}\frac{x-a_{1}}{a_{2}-a_{1}}, & a_{1} \leq x \leq a_{2} \\ \frac{a_{4}-x}{a_{4}-a_{3}}, & a_{3} \leq x \leq a_{4} \\ 0, & \text { elsewhere }\end{cases}
$$




$$
\rho_{\tilde{A}_{I T}}(x)= \begin{cases}\frac{a_{2}-x}{a_{2}-a_{1}^{\circ}}, & a_{1}^{\circ} \leq x \leq a_{2} ; \\ \frac{x-a_{3}}{a_{4}^{\circ}-a_{3}}, & a_{3} \leq x \leq a_{4}^{\circ} ; \\ 1, & \text { elsewhere }\end{cases}
$$

Definition 6 (Smarandache [36]). Let $M$ be a nonempty set. A neutrosophic defined as:

$$
\bar{A}^{N}=\left\{\left\langle x, P_{\bar{A}^{N}}(x), Q_{\bar{A}^{N}}(x), T_{\bar{A}^{N}}(x)\right\rangle: x \in M, P_{\bar{A}^{N}}(x), Q_{\bar{A}^{N}}(x), T_{\bar{A}^{N}}(x) \in\right]^{-} 0,1^{+}[\},
$$

where $P_{\bar{A}^{N}}(x), Q_{\bar{A}^{N}}(x)$, and $T_{\bar{A}^{N}}(x)$ are truth, indeterminacy, and falsity membership functions, respectively, and there is no restriction on the summation of them, so ${ }^{-} 0 \leq P_{\bar{A}^{N}}(x)+$ $Q_{\bar{A}^{N}}(x)+T_{\bar{A}^{N}}(x) \leq 3^{+}$and $]^{-} 0,1^{+}[$is nonstandard unit interval.

Definition 7 (Wang et al. [40]). Let $M$ be a nonempty set. The single valued neutrosophic set $\bar{A}^{N_{S V}}$ of $X$ is defined as:

$$
\bar{A}^{N_{S V}}=\left\{\left\langle x, P_{\bar{A}^{N}}(x), Q_{\bar{A}^{N}}(x), T_{\bar{A}^{N}}(x)\right\rangle: x \in M\right\},
$$

where $P_{\bar{A}^{N}}(x), Q_{\bar{A}^{N}}(x)$, and $T_{\bar{A}^{N}}(x) \in[0,1]$, for each $x \in M$ and $0 \leq P_{\bar{A}^{N}}(x)+Q_{\bar{A}^{N}}(x)+$ $T_{\bar{A}^{N}}(x) \leq 3$.

Definition 8 (Thamaraiselvi and Santhi [39]). Let $\xi_{\tilde{a}}, \psi_{\tilde{a}}, \zeta_{\tilde{a}} \in[0,1]$, and $a_{1}, a_{2}, a_{3}, a_{4} \in \mid r e$ such that $a_{1} \leq a_{2} \leq a_{3} \leq a_{4}$. The single valued trapezoidal neutrosophic number (SVTRN) denoted by $\tilde{a}^{N}=\left\langle\left(a_{1}, a_{2}, a_{3}, a_{4}\right) ; \xi_{\tilde{a}}, \psi_{\tilde{a}}, \zeta_{\tilde{a}}\right\rangle$ is a special neutrosophic set on the real numbers $\Re$, whose truth, indeterminacy, and falsity membership functions are:

$$
\begin{gathered}
\vartheta_{\tilde{a}}(x)= \begin{cases}\xi_{\tilde{a}}\left(\frac{x-a_{1}}{a_{2}-a_{1}}\right), & a_{1} \leq x \leq a_{2} ; \\
\xi_{\tilde{a}}, & a_{2} \leq x \leq a_{3} ; \\
\xi_{\tilde{a}}\left(\frac{a_{4}-x}{a_{4}-a_{3}}\right), & a_{3} \leq x \leq a_{4} ; \\
0, & \text { elsewhere }\end{cases} \\
\rho_{\tilde{a}}(x)= \begin{cases}\frac{a_{2}-x+\psi_{\tilde{a}}\left(x-a_{1}\right)}{a_{2}-a_{1}}, & a_{1} \leq x \leq a_{2} ; \\
\frac{\psi_{\tilde{a}},}{x_{a_{3}+\psi_{\tilde{a}}\left(a_{4}-x\right)},}, & a_{2} \leq x \leq a_{3} ; \\
1, & a_{3} \leq x \leq a_{4} ;\end{cases} \\
\pi_{\tilde{a}}(x)= \begin{cases}\frac{a_{2}-x+\zeta_{\tilde{a}}\left(x-a_{1}\right)}{a_{2}-a_{1}}, & a_{1} \leq x \leq a_{2} ; \\
\zeta_{\tilde{a}}, & a_{2} \leq x \leq a_{3} ; \\
\frac{x-a_{3}+\zeta_{\tilde{a}}\left(a_{4}-x\right)}{a_{4}-a_{3}}, & a_{3} \leq x \leq a_{4} ; \\
1, & \text { elsewhere. }\end{cases}
\end{gathered}
$$

where, $\xi_{\tilde{a}}, \psi_{\tilde{a}}$ and $\zeta_{\tilde{a}}$ are the maximum truth, minimum indeterminacy, and minimum falsity membership degrees, respectively. A single valued trapezoidal neutrosophic number $\tilde{a}^{N}=$ $\left\langle\left(a_{1}, a_{2}, a_{3}, a_{4}\right) ; \xi_{\tilde{a}}, \psi_{\tilde{a}}, \zeta_{\tilde{a}}\right\rangle$ may be expressed on ill-defined quantity about a, which is approximately equal to $\left[a_{2}, a_{3}\right]$.

Definition 9 (Thamaraiselvi and Santhi [39]). Let $\tilde{a}^{N}=\left\langle\left(a_{1}, a_{2}, a_{3}, a_{4}\right), \xi_{\tilde{a}}, \psi_{\tilde{a}}, \zeta_{\tilde{a}}\left\langle\right.\right.$, and $\tilde{b}^{N}=$ $\left\langle\left(b_{1}, b_{2}, b_{3}, b_{4}\right), \xi_{\tilde{b}}, \psi_{\tilde{b}}, \zeta_{\tilde{b}}\langle\right.$, be two single valued trapezoidal neutrosophic numbers and $c \neq 0$, then

(1) $\tilde{a}^{N}(+) \tilde{b}^{N}=\left\langle\left(a_{1}+b_{1}, a_{2}+b_{2}, a_{3}+b_{3}, a_{4}+b_{4}\right) ; \xi_{\tilde{a}} \wedge \xi_{\tilde{b}}, \psi_{\tilde{a}} \vee \psi_{\tilde{b}}, \zeta_{\tilde{a}} \vee \zeta_{\tilde{b}}\right\rangle$, 
(2) $\tilde{a}^{N}(-) \tilde{b}^{N}=\left\langle\left(a_{1}-b_{4}, a_{2}-b_{3}, a_{3}-b_{2}, a_{4}-b_{1}\right) ; \xi_{\tilde{a}} \wedge \xi_{\tilde{b}}, \psi_{\tilde{a}} \vee \psi_{\tilde{b}}, \zeta_{\tilde{a}} \vee \zeta_{\tilde{b}}\right\rangle$,

(3) $\tilde{a}^{N} \otimes \tilde{b}^{N}= \begin{cases}\left\langle\left(a_{1} b_{1}, a_{2} b_{2}, a_{3} b_{3}, a_{4} b_{4}\right) ; \xi_{\tilde{a}} \wedge \xi_{\tilde{b}}, \psi_{\tilde{a}} \vee \psi_{\tilde{b}}, \zeta_{\tilde{a}} \vee \zeta_{\tilde{b}}\right\rangle, & a_{4}>0, b_{4}>0, \\ \left\langle\left(a_{1} b_{4}, a_{2} b_{3}, a_{3} b_{2}, a_{4} b_{1}\right) ; \xi_{\tilde{a}} \wedge \xi_{\tilde{b}}, \psi_{\tilde{a}} \vee \psi_{\tilde{b}}, \zeta_{\tilde{a}} \vee \zeta_{\tilde{b}}\right\rangle, & a_{4}<0, b_{4}>0, \\ \left\langle\left(a_{4} b_{4}, a_{3} b_{3}, a_{2} b_{2}, a_{1} b_{1}\right) ; \xi_{\tilde{a}} \wedge \xi_{\tilde{b}}, \psi_{\tilde{a}} \vee \psi_{\tilde{b}}, \zeta_{\tilde{a}} \vee \zeta_{\tilde{b}}\right\rangle, & a_{4}<0, b_{4}<0,\end{cases}$

(4) $\tilde{\tilde{a}}^{N}= \begin{cases}\left\langle\left(a_{1} / b_{4}, a_{2} / b_{3}, a_{3} / b_{2}, a_{4} / b_{1}\right) ; \xi_{\tilde{a}} \wedge \xi_{\tilde{b}}, \psi_{\tilde{a}} \vee \psi_{\tilde{b}}, \zeta_{\tilde{a}} \vee \zeta_{\tilde{b}}\right\rangle, & a_{4}>0, b_{4}>0, \\ \left\langle\left(a_{4} / b_{4}, a_{3} / b_{3}, a_{2} / b_{2}, a_{1} / b_{1}\right) ; \xi_{\tilde{a}} \wedge \xi_{\tilde{b}}, \psi_{\tilde{a}} \vee \psi_{\tilde{b}}, \zeta_{\tilde{a}} \vee \zeta_{\tilde{b}}\right\rangle, & a_{4}<0, b_{4}>0, \\ \left\langle\left(a_{4} / b_{1}, a_{3} / b_{2}, a_{2} / b_{3}, a_{1} / b_{4}\right) ; \xi_{\tilde{a}} \wedge \xi_{\tilde{b}}, \psi_{\tilde{a}} \vee \psi_{\tilde{b}}, \zeta_{\tilde{a}} \vee \zeta_{\tilde{b}}\right\rangle, & a_{4}<0, b_{4}<0,\end{cases}$

(5) $c \tilde{a}^{N}= \begin{cases}\left\langle\left(c a_{1}, c_{2}, c a_{3}, c a_{4}\right) ; \xi_{\tilde{a}}, \psi_{\tilde{a}}, \zeta_{\tilde{a}}\right\rangle, & c>0, \\ \left\langle\left(c a_{4}, c_{3}, c a_{2}, c a_{1}\right) ; \xi_{\tilde{a}}, \psi_{\tilde{a}}, \zeta_{\tilde{a}}\right\rangle, & c>0,\end{cases}$

(6) $\tilde{a}^{N-1}=\left\langle\left(\frac{1}{a_{4}}, \frac{1}{a_{3}}, \frac{1}{a_{2}}, \frac{1}{a_{1}}\right)\right\rangle ; \xi_{\tilde{a}}, \psi_{\tilde{a}}, \zeta_{\tilde{a}}, \quad \tilde{a}^{N} \neq 0$.

Definition 10. The order relation between two SVTRN numbers is defined as follows:

$$
\left\langle\left(a_{1}, a_{2}, a_{3}, a_{4}\right) ; \xi_{\tilde{q}^{N}}, \psi_{\tilde{q}^{N}}, \zeta_{\tilde{q}^{N}}\right\rangle(=, \geq, \leq) \tilde{b}^{N}=\left\langle\left(b_{1}, b_{2}, b_{3}, b_{4}\right) ; \xi_{\tilde{q}^{N}}, \psi_{\tilde{q}^{N}}, \zeta_{\tilde{q}^{N}}\right\rangle
$$

if and only if

$$
a_{1}\left(\begin{array}{l}
= \\
\leq \\
\geq
\end{array}\right) b_{1}, a_{2}\left(\begin{array}{l}
= \\
\leq \\
\geq
\end{array}\right) b_{2}, a_{3}\left(\begin{array}{l}
= \\
\leq \\
\geq
\end{array}\right) b_{3}, a_{4}\left(\begin{array}{l}
= \\
\leq \\
\geq
\end{array}\right) b_{4},
$$

Definition 11. Let $\tilde{a}^{N}=\left\langle\left(a_{1}, a_{2}, a_{3}, a_{4}\right) ; \xi_{\tilde{a}}, \psi_{\tilde{a}}, \zeta_{\tilde{a}}\right\rangle$ be a SVTRN number. Then we define:

(i) Score function

$$
S\left(\tilde{a}^{N}\right)=(1 / 16) *\left(a_{1}+a_{2}+a_{3}+a_{4}\right) *\left(\vartheta_{\tilde{a}}+\left(1-\rho_{\tilde{a}}\right)+\left(1-\pi_{\tilde{a}}\right)\right)
$$

(ii) Accuracy function

$$
B\left(\tilde{a}^{N}\right)=(1 / 16) *\left(a_{1}+a_{2}+a_{3}+a_{4}\right) *\left(\vartheta_{\tilde{a}}+\left(1-\rho_{\tilde{a}}\right)+\left(1+\pi_{\tilde{a}}\right)\right),
$$

Definition 12. Let $\tilde{a}^{N}, \tilde{b}^{N}$ be any two SVTRN numbers. Then we have:

(i) If $S\left(\tilde{a}^{N}<\left(\tilde{b}^{N}\right), \quad\right.$ then $\tilde{a}^{N}<\tilde{b}^{N}$,

(ii) If $S\left(\tilde{a}^{N}\right)=S \tilde{b}^{N}, \quad$ and if

(1) $B\left(\tilde{a}^{N}\right)<\tilde{\left.\tilde{(} b^{N}\right)}$ then $\tilde{a}^{N}<\tilde{b}^{N}$,

(2) $B\left(\tilde{a}^{N}\right)>B\left(\tilde{b}^{N}\right)$ then $\tilde{a}^{N}>\tilde{b}^{N}$,

(3) $B\left(\tilde{a}^{N}\right)=B\left(\tilde{b}^{N}\right)$ then $\tilde{a}^{N}=\tilde{b}^{N}$, 


\section{Problem formulation and solution concepts}

A fully neutrosophic linear programming (FNLP) problem with $m$ neutrosophic equality constraints and $n$ neutrosophic variables may be formulated as:

$$
\begin{aligned}
& \text { Maximize (or Minimize) } \tilde{Z}^{N}=\tilde{C}^{N} \otimes \tilde{X}^{N} ; \\
& \text { subject to } \\
& \qquad \tilde{A}^{N} \otimes \tilde{X}^{N}=\tilde{B}^{N}
\end{aligned}
$$

where $\tilde{X}^{N}$ is a non-negative SVTRN number, $\tilde{X}^{N}=\left(\tilde{x}_{j}^{N}\right)_{n \times 1}, \tilde{A}^{N}=\left(\tilde{a}_{i j}^{N}\right)_{m \times n}, \tilde{B}^{N}=\left(\tilde{b}_{i}^{N}\right)_{n \times 1}$, and $\tilde{c}_{j}^{N}, \tilde{x}_{j}^{N}, \tilde{a}_{i j}^{N}, \tilde{b}_{i}^{N} \in F(R)$.

Here, $F(R)$ is the set of all single valued trapezoidal neutrosophic numbers.

Definition 13. The Points $\tilde{x}_{j}^{N}$ which satisfies the condition in problem (1) is called a neutrosophic optimization solution of problem (1).

Now, in order to solve the problem (1), let us introduce, a new method to find the neutrosophic optimal slution of FNLP problem in the following steps:

Step 1: Substitutign $\tilde{C}^{N}=\left(\tilde{c}_{j}^{N}\right)_{1 \times n}, \tilde{X}^{N}=\left(\tilde{x}_{j}^{N}\right)_{1 \times n}, \tilde{A}^{N}=\left(\tilde{a}_{i j}^{N}\right)_{m \times n}, \tilde{B}^{N}=\left(\tilde{b}_{i}^{N}\right)_{m \times n}$, the prolbem (1) may be written as:

$$
\text { Maximize (or Minimize) } \tilde{Z}^{N}=\sum_{j=1}^{n} \tilde{c}_{j}^{N} \otimes \tilde{x}_{j}^{N} ;
$$

subject to

$$
\sum_{j=1}^{n} \tilde{a}_{i j}^{N} \otimes \tilde{x}_{j}^{N}=\tilde{b}_{i}^{N}, \quad i=1,2, \ldots, m,
$$

where $\tilde{x}_{j}^{N}$ is a non-negative SVTRN number.

Step 2: Represent all parameters $\tilde{c}_{j}^{N}, \tilde{x}_{j}^{N}, \tilde{a}_{i j}^{N}$, and $\tilde{b}_{i}^{N}$ by the SVTRN numbers $\left\langle\left(p_{j}, q_{j}, r_{j}, s_{j}\right)\right.$; $\left.\alpha_{\tilde{c}_{j}^{N}}, \beta_{\tilde{c}_{j}^{N}}, \delta_{\tilde{c}_{j}^{N}}\right\rangle,\left\langle\left(x_{j}, y_{j}, z_{j}, w_{j}\right) ; \alpha_{\tilde{x}_{j}^{N}}, \beta_{\tilde{x}_{j}^{N}}, \delta_{\tilde{x}_{j}^{N}}\right\rangle,\left\langle\left(a_{i j}, b_{i j}, c_{i j}, d_{i j}\right) ; \alpha_{\tilde{q}^{N}}, \beta_{\tilde{q}^{N}}, \delta_{\tilde{q}^{N}}\right\rangle,\left\langle\left(b_{i}, g_{i}, h_{i}, k_{i}\right) ;\right.$ $\left.\alpha_{\tilde{q}^{N}}, \beta_{\tilde{q}^{N}}, \delta_{\tilde{q}^{N}}\right\rangle$, respectively. Then the problem (2) becomes

$$
\begin{aligned}
\text { Maximize (or Minimize) } \tilde{Z}^{N}= & \sum_{j=1}^{n}\left\langle\left(p_{j}, q_{j}, r_{j}, s_{j}\right) ; \alpha_{\tilde{c}_{j}^{N}}, \beta_{\tilde{c}_{j}^{N}}, \delta_{\tilde{c}_{j}^{N}}\right\rangle \\
& \otimes\left\langle\left(x_{j}, y_{j}, z_{j}, w_{j}\right) ; \alpha_{\tilde{q}_{j}^{N}}, \beta_{\tilde{q}_{j}^{N}}, \delta_{\tilde{q}_{j}^{N}}\right\rangle ;
\end{aligned}
$$

subject to

$$
\begin{aligned}
\sum_{j=1}^{n}\left\langle\left(a_{i j}, b_{i j}, c_{i j}, d_{i j}\right) ; \alpha_{\tilde{a}_{i j}^{N}}, \beta_{\tilde{a}_{i j}^{N}}, \delta_{\tilde{a}_{i j}^{N}}\right\rangle \otimes\left\langle\left(x_{j}, y_{j}, z_{j}, w_{j}\right) ; \alpha_{\tilde{q}^{N}}, \beta_{\tilde{q}^{N}}, \delta_{\tilde{q}^{N}}\right\rangle \\
\leq\left\langle\left(b_{i}, g_{i}, h_{i}, k_{i}\right) ; \alpha_{\tilde{q}^{N}}, \beta_{\tilde{q}^{N}}, \delta_{\tilde{q}^{N}}\right\rangle, \quad i=1,2, \ldots, m,
\end{aligned}
$$

where $\left\langle\left(x_{j}, y_{j}, z_{j}, w_{j}\right) ; \alpha_{\tilde{x}_{j}^{N}}, \beta_{\tilde{x}_{j}^{N}}, \delta_{\tilde{x}_{j}^{N}}\right.$ is a non-negative SVTRN number. 
Step 3: Using the arithmetic operations of SVTRN numbers, the problem (3) may be rewritten as follows:

$$
\begin{aligned}
\text { Maximize (or Minimize) } \tilde{Z}^{N}= & \sum_{j=1}^{n} S\left(\left\langle\left(p_{j}, q_{j}, r_{j}, s_{j}\right) ; \alpha_{\tilde{c}_{j}^{N}}, \beta_{\tilde{c}_{j}^{N}}, \delta_{\tilde{c}_{j}^{N}}\right\rangle\right) \\
& \otimes\left\langle\left(x_{j}, y_{j}, z_{j}, w_{j}\right) ; \alpha_{\tilde{x}_{j}^{N}}, \beta_{\tilde{x}_{j}^{N}}, \delta_{\tilde{x}_{j}^{N}}\right\rangle ;
\end{aligned}
$$

subject to

$$
\begin{aligned}
\sum_{j=1}^{n}\left(\left\langle\left(a_{i j}, b_{i j}, c_{i j}, d_{i j}\right) ; \alpha_{\tilde{a}_{i j}^{N}},\right.\right. & \left.\left.\beta_{\tilde{a}_{i j}^{N}}, \delta_{\tilde{a}_{i j}^{N}}\right\rangle \otimes\left\langle\left(x_{j}, y_{j}, z_{j}, w_{j}\right) ; \alpha_{\tilde{q}^{N}}, \beta_{\tilde{q}^{N}}, \delta_{\tilde{q}^{N}}\right\rangle\right) \\
& \leq\left\langle\left(b_{i}, g_{i}, h_{i}, k_{i}\right) ; \alpha_{\tilde{q}^{N}}, \beta_{\tilde{q}^{N}}, \delta_{\tilde{q}^{N}}\right\rangle \\
& i=1,2, \ldots, m ; y_{j}-x_{j} \geq 0, w_{j}-z_{j} \geq 0, \forall j=1,2, \ldots, n .
\end{aligned}
$$

Step 4: Find the optimal solution $x_{j}, y_{j}, z_{j}$, and $w_{j}$ by solving the problem (4).

Step 5: Find the neutrosophic solution by putting the values of $x_{j}, y_{j}, z_{j}$, and $w_{j}$ in $\tilde{x}_{j}^{N}=$ $\left\langle\left(x_{j}, y_{j}, z_{j}, w_{j}\right) ; \alpha_{\tilde{x}_{j}^{N}}, \beta_{\tilde{x}_{j}^{N}}, \delta_{\tilde{x}_{j}^{N}}\right\rangle$.

Step 6: Find the neutrosophic optimum value by putting $\tilde{x}_{j}^{N}$ into $\sum_{j=1}^{n} \tilde{c}_{j}^{N} \otimes \tilde{x}_{j}^{N}$.

\section{Stock portfolio investment problem}

In this section, the stock portfolio investment problem introduced by Yin [41] is studied in the neutrosophic environment. Before introducing the problem-formulation, some of required assumptions and notation are described.

\subsection{Assumptions}

In the stock portfolio problem, the following assumptions are used:

- The expected rate of return and risk loss rate are evaluated by investors.

- Securities are indefinite and may be divided.

- No need to pay the transaction costs in the course of transaction.

- Assumptions of non-satisfaction and avoiding tisk are obeyed by investors.

- Short selling operation is not allowed.

- Interest rate of the bank is unchanged for investors during investment period.

\subsection{Notation}

In the stock problem, the following notion can be used:

\section{Index:}

$j$ : Risk securities.

\section{Decision variable:}

$x_{j}$ : Proportion of funds invested in the secondary securities.

$x_{0}$ : Proportion of the total amount of investment in the investment period.

$x$ : Expected return rate of an investment combination introduced by investors. 


\section{Parameters:}

$A$ : Rate of risked return.

$r_{j}$ : Expected return rate.

$B$ : Portfolio risk amount.

$r_{0}$ : Interst rate of the bank.

Hence, the problem can be formulated as follows:

$$
\operatorname{Max}) \tilde{R}^{N}=\tilde{r}_{0}^{N} \otimes \sum_{j=1}^{n} \tilde{r}_{j}^{N} \otimes x_{j}
$$

subject to

$$
\begin{gathered}
\tilde{A}^{N} \otimes x \leq \tilde{B}^{N} ; \\
x_{0}+\sum_{j=1}^{n} x_{j}=1, \\
x_{j} \leq 0, \quad j=1,2, \ldots, n .
\end{gathered}
$$

where, $\tilde{A}^{N}=\left(\tilde{a}_{i j}^{N}\right)_{m \times n}, \tilde{B}^{N}=\left(\tilde{b}_{1}^{N}, \ldots, \tilde{b}_{m}^{N}\right)^{T}, \tilde{r}^{N}=\left(\tilde{r}_{1}^{N}, \ldots, \tilde{r}_{n}^{N}\right)^{T}$, and $X=\left(x_{1}, \ldots, x_{n}\right)^{T}$.

Example 1. Consider the following FNLPproblem

$$
\begin{aligned}
& \max \tilde{Z}^{N}=\langle(3,5,6,8) ; 0.6,0.5,0.4\rangle \otimes\langle(5,8,10,14) ; 0.3,0.6,0.6\rangle \oplus \tilde{x}_{2}^{N} \\
& \text { subject to } \\
& \quad\langle(3,5,6,8) ; 0.6,0.5,0.4)\rangle \otimes \tilde{x}_{1}^{N} \oplus\langle(5,8,10,14) ; 0.3,0.6,0.6\rangle \otimes \tilde{x}_{2}^{N} \\
& \quad=\langle(16,18,22,30) ; 0.8,0.2,0.3\rangle, \\
& \quad\left\langle(5,8,110,14) ; 0.3,0.6,0.6 \otimes \tilde{x}_{1}^{N} \oplus\langle(3,5,6,8) ; 0.6,0.5,0.4)\right\rangle \otimes \tilde{x}_{2}^{N} \\
& \quad=\langle(13,15,18,24) ; 0.8,0.2,0.3\rangle,
\end{aligned}
$$

Also, $\tilde{x}_{1}^{N}$ and $\tilde{x}_{2}^{N}$ are non-negative SVTRN numbers.

Let $\tilde{x}_{1}^{N}=\left\langle\left(x_{1}, y_{1}, z_{1}, w_{1}\right) ; 0.8,0.2,0.3\right\rangle, \tilde{x}_{2}^{N}=\left\langle\left(x_{2}, y_{2}, z_{2}, w_{2}\right) ; 0.8,0.2,0.3\right\rangle$ be non-negative SVTRN numbers. By using the step 2, the above problem becomes

$\max \tilde{Z}^{N}=\langle(3,5,6,8) ; 0.6,0.5,0.4\rangle \otimes\left\langle\left(x_{1}, y_{1}, z_{1}, w_{1}\right) ; 0.8,0.2,0.3\right\rangle \oplus\langle(5,8,10,14) ; 0.3,0.6,0.6\rangle$ $\otimes\left\langle\left(x_{2}, y_{2}, z_{2}, w_{2}\right) ; 0.8,0.2,0.3\right\rangle$

subject to

$$
\begin{aligned}
& \left\langle\left(x_{1}, y_{1}, z_{1}, w_{1}\right) ; 0.8,0.2,0.3\right\rangle \oplus 2\left\langle\left(x_{2}, y_{2}, z_{2}, w_{2}\right) ; 0.8,0.2,0.3\right\rangle=\langle(16,18,22,30) ; 0.8,0.2,0.3\rangle, \\
& \left\langle\left(x_{1}, y_{1}, z_{1}, w_{1}\right) ; 0.8,0.2,0.3\right\rangle \oplus\left\langle\left(x_{2}, y_{2}, z_{2}, w_{2}\right) ; 0.8,0.2,0.3\right\rangle=\langle(13,15,18,24) ; 0.8,0.2,0.3\rangle .
\end{aligned}
$$

Moreover, $\left\langle\left(x_{1}, y_{1}, z_{1}, w_{1}\right) ; 0.8,0.2,0.3\right\rangle$, and $\left\langle\left(x_{2}, y_{2}, z_{2}, w_{2}\right) ; 0.8,0.2,0.3\right\rangle$ are non-negative $S V T R N$ numbers. By using step 3, the above problem can be written as:

$$
\max Z=\left(\frac{1}{16}\right)\left(5.1 x_{1}+8.5 y_{1}+10.2 z_{1}+13.6 w_{1}+5.5 x_{2}+8.8 y_{2}+11 z_{2}+15.4 w_{2}\right)
$$

subject to

$$
\begin{array}{ll}
x_{1}+2 x_{2}=16 ; & y_{1}+2 y_{2}=18 ; \\
z_{1}+2 z_{2}=22 ; & w_{1}+2 w_{2}=30 ; \\
2 x_{1}+x_{2}=13 ; & 2 y_{1}+y_{2}=18 ; \\
2 z_{1}+z_{2}=18 ; & 2 w_{1}+w_{2}=24 \\
y_{1}-x_{1} \geq 0, & w_{1}-z_{1} \geq 0 ; \\
y_{2}-x_{2} \geq 0, & w_{2}-z_{2} \geq 0
\end{array}
$$


By using the MATLAB, we obtain the optimal solution of the problem as follows:

$$
\begin{array}{llll}
x_{1}^{*}=3.3, & y_{1}^{*}=4, & z_{1}^{*}=4.6, & w_{1}^{*}=6 ; \\
x_{2}^{*}=6.3, & y_{2}^{*}=7, & z_{2}^{*}=8.3, & w_{2}^{*}=12 .
\end{array}
$$

By using step 5, the neutrosophic solution is given by

$$
\tilde{x}_{1}^{N}=\langle(3.3,4,4.6,6) ; 0.8,0.2,0.3\rangle,
$$

and

$$
\tilde{x}_{2}^{N}=\langle(6.3,7,8.3,12) ; 0.8,0.2,0.3\rangle
$$

and hence the neutrosophic optimum value is

$$
\tilde{Z}^{N}=\langle(41.4,78,119,220.8) ; 0.3,0.6,0.6\rangle .
$$

Example 2. Consider the data of the expected return rate the risk loss rate and the risk coefficient of two stocks listed as follows:

Expected return rate \%

$$
\tilde{r}_{1}^{N}=\left\langle(1,2,3,4 ; 0.1,0.9,1\rangle, \quad \tilde{r}_{2}^{N}=\langle(0,2,3,5 ; 0.1,0.9,0.9\rangle .\right.
$$

Risk loss rate $\%$

$$
\begin{array}{llrl}
\tilde{a}_{11}^{N}=\langle(1,2,4,6 ; 0.1,0.9,0.9\rangle, & & \tilde{a}_{12}^{N}=\langle(1,3,4,5 ; 0.1,0.8,0.1\rangle . \\
\tilde{a}_{21}^{N}=\langle(2,3,4,6 ; 0.1,0.8,1\rangle, & & \tilde{a}_{22}^{N}=\langle(3,4,6,7 ; 0.1,0.8,0.9\rangle .
\end{array}
$$

risk coefficient $\%$

$$
\tilde{b}_{1}^{N}=\left\langle(0,2,3,4 ; 0.03,0.95,0.98\rangle, \quad \tilde{b}_{2}^{N}=\langle(1,2,3,5 ; 0.03,0.98,0.95\rangle,\right.
$$

for the return rate $\tilde{r}_{0}^{N}=\langle(0,1,2,3,4 ; 0.1,0.9,1\rangle$.

According to problem (5) and by using the score function in definition (11), we have

$$
\begin{aligned}
& \text { MaxR }=0.075 x_{0}+0.125 x_{1}+0.1875 x_{2} ; \\
& \text { subject to } \\
& \quad 0.24375 x_{1}+0.235 x_{2} \leq 0.056 \\
& 0.26250 x_{1}+0.500 x_{2} \leq 0.0625 \\
& x_{0}+x_{1}+x_{2}=1, \quad j=0,1,2 . \\
& \quad x_{j} \leq 0, \quad j \quad
\end{aligned}
$$

The optimal solution is $\left(x_{0}^{*}, x_{1}^{*}, x_{2}^{*}\right)=(0.8750,0,0.125)$, and the corresponding optimum value is $R=0.0891$. Hence the neutrosophic optimum value is $\tilde{R}^{N}=\langle 0,1.125,3,3.25 ; 0.1,0.9,0.9\rangle$.

\section{Conclusions}

In this paper, we proposed a new method to find the neutrosophic optimal solution of the linear programming problem in neutrosophic environment with equality constraints. The advantage of this approach is that neutrosophic linear programming allows the DM to deal with the situation under uncertainty realistically. In addition, it is solved easily by using on neutrosophic theory. The study under uncertainty makes the investment portfolio more realistic and practice to describe the expected return rate, and also risk loss rate. The advantages of the method for the investors are: the ability for choosing the risk coefficient so as to achieve higher expected returns, and also determining his/ her strategies for selecting the portfolios. 


\section{Acknowledgements}

The authors would like to thank the Editor-in- Chief and anonymous referee for his/her suggestive and hepful comments that have led to an improvement both in the quality a clarity of the paper and also to the Deanship of informative research at Qassim University as well as VIT Bhopal University.

\section{References}

[1] Abdel-Basset, M., Gunasekaran, M., Mohamed, M. and Smarandache, F. (2019). A novel method for solving the fully neutrosophic linear programming problem. Neural Computing and Applications, 31(5), 1595-1605. doi: 10.1007/s00521-018-3404-6

[2] Allahviranloo, T., Lotfi, F. H., Kiasary, M. K., Kiani, N. A. and Alizadeh, L. (2008). Solving fully fuzzy linear programming problem by the ranking function. $A p$ plied Mathematical Sciences, 2(1), 19-32. http://www.m-hikari.com/ams/ams-password-2008/ ams-password1-4-2008/lotfiAMS1-4-2008-2.pdf

[3] Atanassov, T. K. (1986). Intuitionistic fuzzy set. Fuzzy Sets and Systems, 20(1), 87-96. doi: 10.1016/S0165-0114(86)80034-3

[4] Bellman, R. E. and Zadeh, L. A. (1970). Decision making in a fuzzy environment. Management Science, 17(4), 141-164. doi: 10.1287/mnsc.17.4.B141

[5] Buckley, J. J. (1989). Solving possibilistic linear programming problems. Fuzzy Sets and Systems, 31(3), 329-341. doi: 10.1016/0165-0114(89)90204-2

[6] Campos, L. and Verdegay, J.L. (1989). Linear programming problems and ranking of fuzzy numbers. Fuzzy Sets and Systems, 32(1), 1-11. doi: 10.1016/0165-0114(89)90084-5

[7] Darehmiraki, M. (2020). A solution for the neutrosophic linear programming problem with a new ranking function. In Smarandache, F. and Abdel-Basset, M. (Eds.) Optimization Theory Based on Neutrosophic and Plithogenic Sets (pp. 235-259). Elsevier. doi: 10.1016/B978-0-12-819670$0.00011-1$

[8] Das, S. K. (2017). Modified method for solving fully fuzzy linear programming problem with triangular fuzzy numbers. International Journal of Research in Industrial Engineering, 6(4), 293311. doi: 10.22105/RIEJ.2017.101594.1024

[9] Dutta, D. and Kumar, P. (2015). Application of fuzzy goal programming approach to multiobjective linear fractional inventory model. International Journal of Systems Science, 46(12), 2269-2278. doi: 10.1080/00207721.2013.860639

[10] Ebrahimnejad, A., Nasseri, S. H., Lotfi, F. H. and Soltanifar, M. (2010). A primal-dual method for linear programming problems with fuzzy variables. European Journal of Industrial Engineering, 4(2), 189-209. doi: 10.1504/EJIE.2010.031077

[11] Ezzati, R., Khorram, R. and Enayati, R., A. (2015). A new algorithm to solve fully fuzzy linear programming problems using the MOLP problem. Applied Mathematical Modelling, 39(12), 31833193. doi: 10.1016/j. apm.2013.03.014

[12] Ganesan, K. and Veeramani, P. (2006). Fuzzy linear programs with trapezoidal fuzzy numbers. Annals of Operational Research, 143(1), 305-315. doi: 10.1007/s10479-006-7390-1

[13] Gao, J. and Liu, H. (2017). A risk-free protection index model for portfolio selection with entropy constraints under an uncertainty framework. Entropy, 19(2), 80. doi: 10.3390/e19020080

[14] Gong, Z., Zhao, W. and Liu, K. (2018). A straightforward approach for solving fully fuzzy linear programming problem with LR-type fuzzy numbers. Journal of the Operations Research Society of Japan, 61(2), 172-185. doi: 10.15807/jorsj.61.172

[15] Hashemi, S. M., Modarres, M., Nasrabadi, E. and Nasrabadi, M. M. (2006). Fully fuzzified linear programming solution and duality. Journal of Intelligence and Fuzzy Systems, 17(3), 253-261. https://content.iospress.com/articles/journal-of-intelligent-and-fuzzy-systems/ ifs00295

[16] Huang, X. and Ying, H. (2013). Risk index based models for portfolio adjusting problem with returns subject to expert's evaluations. Economic Model, 30, 61-66. doi: 10.1016/j.econmod.2012.09.032 
[17] Hosseinzadeh, A. and Edalatpanah, A. S. (2016). A new approach for solving fully fuzzy linear programming by using the Lexicography method. Advances in Fuzzy Systems, 1-6. doi: 10.1155/2016/ 1538496

[18] Hussein, A.-N., Mohamed, M., Abdel-Basetm M. and Smarandache, F. (2018). Neutrosophic linear programming problems. In Smarandache, F. and Abdel-Basset, M. (Eds.) Neutrosophic Operations Research (pp. 1-15). Elsevier. doi: 10.1007/978-3-030-57197-9

[19] Ishibuchi, H. and Tanaka, H. (1990). Multiobjective programming in optimization of the interval objective function. European Journal of Operational Research, 48(2), 219-225. doi: 10.1016/03772217(90)90375-L

[20] Jianqiang, W. and Zhong, Z. (2009). Aggregation operators on intuitionistic trapezoidal fuzzy number and its application to multi-criteria decision making problems. Journal of Systems Engineering and Electronics, 20(2), 321-326. https://ieeexplore.ieee.org/document/6074660

[21] Kaufmann, A. and Gupta, M. M. (1988). Fuzzy Mathematical Models in Engineering and Management Science. New York: Elsevier.

[22] Khalifa, H. A. (2019). Utilizing a new approach for solving fully fuzzy linear programming problems. Croatian Operational Research Review, 10(2), 337-344. doi: 10.17535/crorr.2019.0027

[23] Khalifa, H. A., Kumar, P., and Smarandache, F. (2020). On optimizing neutrosophic complex programming using lexicographic order. Neutrosophic Sets and Systems, 32, 330-343. doi: $10.5281 /$ zenodo. 3723173

[24] Kumar, P. (2019). An inventory planning problem for time-varying linear demand and parabolic holding cost with salvage value. Croatian Operational Research Review, 10(2), 187-199. doi: 10.17535 /crorr.2019.0017

[25] Kumar, A., Kaur, J. and Singh, P. (2011). A new method for solving fully fuzzy linear programming problems. Applied Mathematical Modelling, 35(2), 817-823. doi: 10.1016/j.apm.2010.07.037

[26] Li, X. and Shi, K. (1998). Fuzzy linear programming problems with interval-value fuzzy coefficients. Journal of Fuzzy Mathematics, 6(2), 327-332.

[27] Liu, Y. and Qin, Z. (2012). Mean semi-absolute deviation model for uncertain portfolio optimization problem. Journal of Uncertain Systems, 6, 299-307. http://www . worldacademicunion.com/ journal/jus/jusVol06No4paper09.pdf

[28] Maleki, H. R., Tata, M. and Mashinchi, M. (2000). Linear programming with fuzzy variables. Fuzzy Sets and Systems, 109, 21-33. doi: 10.1016/S0165-0114(98)00066-9

[29] Maleki, H. R. (2002). Ranking functions and their applications to fuzzy linear programming. Far East of Mathematical Science, 4, 283-301.

[30] Markowitz, H. (1952). Portfolio selection. Journal of Finance, 7, 77-91. doi: 10.1111/j.15406261.1952.tb01525.x

[31] Nafei, A., Arif, M., Yuan, W. and Nasseri, H. (2020). A new method for solving interval neutrosophic linear programming problems. Acta Polytechnica Hungarica, 1-17. doi: 10.35378 /gujs.689125

[32] Prameela, K. U. and Kumar, P. (2020). Perceptionization of FM/FD/1 queuing model under various fuzzy numbers. Croatian Operational Research Review, 11(1), 135-144. doi: 10.17535/crorr.2020.0011

[33] Rajarajeswari, P. and Sahaya S. A. (2014). Solving a fully fuzzy linear programming problem by ranking. International Journal of Mathematics Trends and Technology, 9(2), 159-164. doi: 10.14445/22315373/IJMTT-V9P519

[34] Shamooshaki, M. M., Hosseinzadeh, A. and Edalatpanah, S. A. (2015). A new method for solving fully fuzzy linear programming problems by using the Lexicography method. Applied and Computational Mathematics, 4(1-2), 1-3. doi: 10.11648/j.acm.s.2015040102.11

[35] Shaocheng, T. (1994). Interval number and fuzzy number linear programmings. Fuzzy Sets and Systems, 66, 301-306. doi:10.1016/0165-0114(94)90097-3

[36] Smarandache, F. (1998). A Unifying Field in Logics, Neutrosophic: Neutrosophic Probability, Set and Logic. American Research Press, Rehoboth, NM, USA.

[37] Sudha, A. S. and Karpagamani, V. (2014). Solving fully fuzzy linear programming problem using trapezoidal ranking function. Journal of Global Research in Mathematical Archives, 2(6), 21-26.

[38] Tanaka, H., Okuda, T. and Asai, K. (1974). On fuzzy mathematical programming. Journal of Cybernetics, 3, 37-46. doi: 10.1080/01969727308545912 
[39] Thamaraiselvi, A. and Santhi, R. (2016). A new approach for optimization of real life transportation problem in neutrosophic environment. Mathematical Problem in Engineering. doi: $10.1155 / 2016 / 5950747$

[40] Wang, H., Smarandache, F., Zhang, Q. Y. and Sunderraman, R. (2010). Single valued neutrosophic sets. Multispace and Multistructure, 4, 410-413.

[41] Yin, D. (2018). Application of interval valued fuzzy linear programming for stock portfolio optimization. Applied Mathematics, 9, 101-113. doi: 10.4236/am.2018.92007

[42] Zadeh, L. A. (1965). Fuzzy sets. Information Control, 8, 338-353. doi: 10.1016/S00199958(65)90241-X

[43] Zhao, R., Govind, R. and Fan, G. (1992). The complete decision set of the generalized symmetrical fuzzy linear programming problem. Fuzzy Sets and Systems, 51, 53-65. doi: 10.1016/01650114(92)90075-F

[44] Zhong, Q., Zhang Y. and Guangyuan, W. (1994). On fuzzy random linear programming. Fuzzy Sets and Systems, 65, 31-49. doi: 10.1016/0165-0114(94)90245-3

[45] Zhu, Y. (2010). Uncertain optimal control with application to a portfolio selection model. Cybernetic System, 41, 535-547. doi: 10.1080/01969722.2010.511552

[46] Zimmermann, H-J. (1978). Fuzzy programming and linear programming with several objective functions. Fuzzy Sets and Systems, 1, 45-55. doi: 10.1016/0165-0114(78)90031-3

[47] Zimmermann, H.-J. (2001). Fuzzy Set Theory and its Applications - 4th Ed., Kluwer Academic Publishers. 